\title{
Laboratory preparedness: building a cornerstone for global surveillance
}

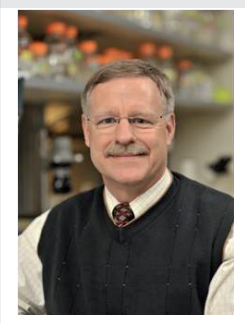

“The drivers of disease emergence, while not completely understood, have enough clarity to make sound predictions that pandemic H1N1 influenza will not be the last emerging infectious disease of this new decade."

\section{Terry F McElwain}

School for Global Animal Health and Washington Animal Disease Diagnostic Laboratory, College of Veterinary Medicine, Washington State University, Pullman, WA 99164-7040, USA = Tel. : +1 5093355861 = Fax: +1 5093356328 =tfm@vetmed.wsu.edu

Pandemic H1N1 influenza is but the latest in what has been a continuum of infectious disease outbreaks. As we approach the beginning of the end of the H1N1 global influenza pandemic, the need for improved disease surveillance that would allow earlier recognition of an emergent disease has been, and will continue to be, discussed [1-3]. The human, social and economic impact of pandemic H1N1 is a story to be told at a later time. However, a recent National Academy of Sciences (NAS) Institute of Medicine (IOM) and National Research Council (NRC) report, Sustaining Global Surveillance and Response to Emerging Zoonotic Diseases, claims that the economic impact of novel diseases over the last decade alone has exceeded US $\$ 200$ billion [4]. The drivers of disease emergence, while not completely understood, have enough clarity to make sound predictions that pandemic H1N1 influenza will not be the last emerging infectious disease of this new decade. Are we prepared globally for the curtain to rise on the next act?

\section{Building on success}

The joint IOM/NRC report on global disease surveillance outlined a broad range of recommendations around establishing and sustaining a global surveillance system, including examples of ways in which it might be financed. In considering the challenges of creating a global surveillance system, particularly when the socalled 'hotspots' [5] are located in regions of the world crippled by poverty, war and famine, one can easily become discouraged. Yet, I would suggest that there are success stories upon which we can continue to build in the future, and which justify continued investment. The threat of Eurasian strain $\mathrm{H} 5 \mathrm{~N} 1$ avian influenza brought to bear significant resources in both underdeveloped nations and the most highly developed countries to establish or expand on existing frameworks for disease surveillance and response. Many developed nations, through the WHO, United Nations Food and Agriculture Organization (FAO), World Bank, donor agencies and others, provided resources and guidance for this process in resourcelimited nations, and the world embarked on surveillance programs that targeted early recognition and reporting of this threat to global health. In laboratories, preparation for $\mathrm{H} 5 \mathrm{~N} 1$ influenza surveillance and diagnosis included guidance and funding to ensure the appropriate biocontainment facilities, implementing rapid $\mathrm{H} 5 \mathrm{~N} 1$-specific assays, providing equipment to run these assays, training staff to perform and interpret results and, importantly, establishing operational procedures and lines of communication among stakeholders at all levels in the animal and human health communities. At the clinical or field level, procedures for sample collection and submission, data entry, sample identification and database development were implemented. The challenges of sharing information in a global database were recognized and addressed in a broadly acceptable manner [6,7].

While Eurasian $\mathrm{H} 5 \mathrm{~N} 1$ avian influenza cases continue to occur in both animals and humans, primarily in Asia, and warrants close monitoring, the foundation for global influenza surveillance was established with protocols that could be used to respond to an outbreak of any novel influenza strain. In the animal health realm, investment into that foundation enabled a more rapid response to pandemic H1N1 influenza at both the laboratory and clinical levels. A salient example is the speed with which assays were developed and

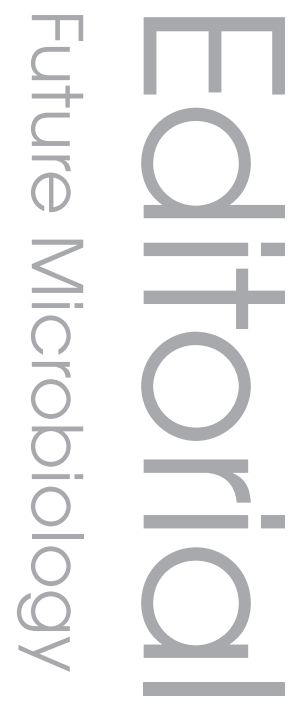

66...the foundation for global influenza surveillance was established with protocols that could be used to respond to an outbreak of any novel influenza strain."

\section{future
Medicine}


"Information and data

flow from the local to

regional, national and international levels - and back - should be seamless to permit rapid, integrated data analysis." deployed worldwide, including in the USA. Early sharing of virus isolates between human and animal health sectors enabled rapid analytical validation of a sensitive and specific realtime PCR assay for swine by May 2009 that was quickly deployed, along with an approved testing algorithm, in 36 animal health testing laboratories across the USA [101]. That testing algorithm was rapidly adopted by Offlu, the joint World Organization for Animal Health (OIE)/FAO network of expertise on animal influenza [102]. Concurrently, the CDC refined an H1N1 real-time PCR assay for use with human samples, with justifiable differences from the animal assay based both on the viral strains that might be encountered in the respective target populations and to avoid overlap of critical reagents that would cripple testing nationwide if a manufacturing or quality failure resulted in a reagent shortage. The speed with which this happened in animal health laboratories in the USA simply could not have been achieved without prior investment in a national disease surveillance and laboratory infrastructure. That infrastructure is coordinated at the laboratory level through the National Animal Health Laboratory Network, created in 2002 with funding to address bioterrorist threats [103]. The investment has earned unexpected dividends, and is a salient example of how a coordinated laboratory network based on sound principles of laboratory and network function can be used to rapidly confront surveillance for a new disease.

\section{An integrated global laboratory network:} steps toward the future

To address the need for global laboratory infrastructure - only one of many critical components of a global surveillance system - the IOM/NRC report recommended promotion and establishment of an international laboratory working group charged with designing a global laboratory network plan for zoonotic disease surveillance. The report emphasized that such a plan must "integrate to the maximum extent possible animal and human health components, and that a long term coordinating body, in consultation with WHO, FAO and the OIE, should implement the global laboratory network plan, sustain it, and continuously assess its performance for zoonotic disease surveillance." Proposed steps for developing the plan included conducting an inventory of the current global capacity for diagnosis and reporting of zoonotic diseases in both human and animal health laboratories. While this step might be viewed as a rather mundane task, a comprehensive inventory of global public and animal health diagnostic laboratory expertise does not exist and would provide a basis for designing a global laboratory network structure. Importantly, it would also help establish priorities for investment in additional laboratory infrastructure. Indeed, the report recommended that based on this inventory, the working group could design the optimal laboratory network structure utilizing existing, or where needed developing new regional reference laboratories in high-risk regions capable of a broad range of laboratory diagnostics to identify known and newly emergent agents. There are 'hidden gems' of public and animal health laboratory expertise and infrastructure in some of the poorest of nations, which with relatively limited investment could become much-needed regional reference laboratories with broader expertise. The proposed laboratory network design would also address sentinel laboratory needs in high-risk regions for normative diagnosis and early recognition of emergent syndromes. As the report emphasized, an environment for integrated animal and public health zoonotic disease diagnosis and reporting is essential for sound resource investment in laboratory infrastructure. There is no sound scientific reason for duplicating reference laboratory facilities for both health sectors when the vast majority of emergent infectious diseases of humans are caused by zoonotic agents, and when assays for identifying those agents require identical equipment and technical expertise. There are valid reasons to separate specific testing protocols such as virus isolation when recombination in the laboratory may result in highly virulent, laboratory-based variants [8]. However, good laboratory practice, verified through inspection and audit procedures and, where needed, effective separation of animal and human health testing within the same facility, can provide safeguards against laboratory accidents. The result would be a single center of excellence in laboratory diagnostics to serve all sectors (a goal worthy of consideration in developed nations). Information and data flow from the local to regional, national and international levels - and back - should be seamless to permit rapid, integrated data analysis.

Creating and sustaining a global laboratory network is a mighty undertaking. However, if a broadly acceptable plan could be developed, what would implementation of the plan require, 
and how could it be sustained? (Financing global surveillance, including laboratory components, is addressed separately in the report. The reader is referred to recommendation $2-1$ and chapter 6 of the report.) The NAS report suggested that an international coordinating body could be responsible for both steps. An example, but neither the only nor the perfect one, is the United Nations System Influenza Coordinator's office (UNSIC) [104]. A UNSIC-like office could bring together intergovernmental organizations and international animal and human health laboratory communities into a global body with responsibility for oversight and monitoring of development and performance of laboratories in the network.

\section{Getting a start}

Is the global heath community ready for such an undertaking? The threat of bioterrorism and the recognition of zoonotic disease threats of the past decade have given the animal and public health laboratory communities a badly needed boost. Basic and translational science and informatics have provided us with unprecedented knowledge of the microbial world at the genetic and proteomic level, and the tools to take advantage of this knowledge in disease diagnosis and data analysis. I would argue that the public is not only behind us, but indeed is waiting for us to lead the way. We have momentum. As always, the 'devil is in the details'. The first recommended action in the NAS report - establishing and convening an international laboratory working group to look closely at the details of a global, integrated laboratory network plan - is an important and inexpensive first step. Much could be accomplished with a relatively small working group of laboratory professionals with experience in laboratory network development meeting for an intensive week of deliberations. The cost is small for this first step, and the stakes are high. It is a step well worth taking.

\section{Financial \& competing interests disclosure \\ TF McElwain is a Professor in the School for Global Animal Health and Executive Director of the Washington Animal Disease Diagnostic Laboratory. He served as a committee member and is a co-author on the Institute of Medicine and National Research Council report Sustaining Global Surveillance and Response to Emerging Zoonotic Diseases. The author has no other relevant affiliations or financial involvement with any organization or entity with a financial interest in or financial conflict with the sub- ject matter or materials discussed in the manuscript apart from those disclosed. \\ No writing assistance was utilized in the production of this manuscript.}

\section{Bibliography}

1. Fidler DP: H1N1 after action review: learning from the unexpected, the success and the fear. Future Microbiol. 4(7), 767-769 (2009).

2. Levi J, Inglesby TV, Segal LM, Vinter S: Pandemic flu preparedness (issue brief). Trust for America's Health, Robert Wood Johnson Foundation, Washington, DC, USA, 1-24 (2009).

3. Vargas-Parada L: H1N1: a Mexican perspective. Cell 139(7), 1203-1205 (2009).

4. Institute of Medicine and National Research Council: Sustaining Global Surveillance and Response to Emerging Zoonotic Diseases. Keusch GT, Pappaioanou M, Gonzalez MC, Scott KA, Tsai P (Eds). The National Academies Press, Washington, DC, USA, 1-16 (2009).

5. Jones KE, Patel NG, Levy MA et al.: Global trends in emerging infectious diseases. Nature 451(7181), 990-993 (2008).
6. Bao Y, Bolotov P, Dernovoy D et al.: The Influenza Virus Resource at the National Center for Biotechnology Information. J. Virol. 82(2), 596-601 (2008).

7. Fidler DP: Influenza virus samples, international law, and global health diplomacy. Emerg. Infect. Dis. 14(1), 88-94 (2008).

8. WHO: WHO Manual on influenza diagnosis and surveillance. Webster RG, Krause S, WHO Animal Influenza Network (Eds). WHO, Geneva, Switzerland, 27 (2002).

\section{Websites}

101. USDA, APHIS, Animal Health, National Animal Health Laboratory Network, Laboratory list, Swine Influenza Virus. www.aphis.usda.gov/animal_health/nahln/ downloads/siv_lab_list.pdf. (Accessed 4 February 2010)

102. Offlu interim guidance on detecting pandemic H1N1 2009 in pigs: laboratory testing algorithm.
www.offlu.net/OFFLU\%20Site/OFFLU_ SIV_Surveillance_Testing_Algorithm.pdf. (Accessed 4 February 2010)

103. USDA, APHIS, Animal Health, National Animal Health Laboratory Network. www.aphis.usda.gov/animal_health/nahln/. (Accessed 4 February 2010)

104. United Nations System Influenza Coordinator. www.undg.org/index.cfm? $\mathrm{P}=21$. (Accessed 4 February 2010)

\section{Affiliation}

- Terry F McElwain

School for Global Animal Health and Washington Animal Disease Diagnostic Laboratory, College of Veterinary Medicine, Washington State University, Pullman, WA 99164-7040, USA Tel.: +1 5093355861

Fax: +15093356328

tfm@vetmed.wsu.edu 